\title{
The solar photospheric nitrogen abundance
}

\section{Analysis of atomic transitions with 3D and 1D model atmospheres}

\author{
E. Caffau ${ }^{1}$, E. Maiorca ${ }^{2}$, P. Bonifacio ${ }^{3,1,4}$, R. Faraggiana ${ }^{5}$, M. Steffen ${ }^{6}$, H.-G. Ludwig ${ }^{3,1}$, I. Kamp ${ }^{7}$, and M. Busso ${ }^{2,8}$ \\ 1 GEPI, Observatoire de Paris, CNRS, Université Paris Diderot, 92195 Meudon Cedex, France \\ e-mail: Elisabetta.Caffau@obspm.fr \\ 2 Department of Physics, University of Perugia, via Pascoli, 06123 Perugia, Italy \\ CIFIST Marie Curie Excellence Team, France \\ ${ }^{4}$ Istituto Nazionale di Astrofisica, Osservatorio Astronomico di Trieste, via Tiepolo 11, 34143 Trieste, Italy \\ 5 Dipartimento di Astronomia, Università degli Studi di Trieste, via G.B. Tiepolo 11, 34143 Trieste, Italy \\ 6 Astrophysikalisches Institut Potsdam, An der Sternwarte 16, 14482 Potsdam, Germany \\ 7 Kapteyn Astronomical Institute, Postbus 800, 9700 AV Groningen, The Netherlands \\ 8 Istituto Nazionale di Fisica Nucleare, section of Perugia, via Pascoli, 06123 Perugia, Italy
}

Received 26 August 2008 / Accepted 28 January 2009

\section{ABSTRACT}

\begin{abstract}
Context. In recent years, the solar chemical abundances have been studied in considerable detail because of discrepant values of solar metallicity inferred from different indicators, i.e., on the one hand, the "sub-solar" photospheric abundances resulting from spectroscopic chemical composition analyses with the aid of 3D hydrodynamical models of the solar atmosphere, and, on the other hand, the high metallicity inferred by helioseismology.

Aims. After investigating the solar oxygen abundance using a CO5 BOLD 3D hydrodynamical solar model in previous work, we undertake a similar approach studying the solar abundance of nitrogen, since this element accounts for a significant fraction of the overall solar metallicity, $Z$.

Methods. We used a selection of atomic spectral lines to determine the solar nitrogen abundance, relying mainly on equivalent width measurements in the literature. We investigate the influence on the abundance analysis, of both deviations from local thermodynamic equilibrium ("NLTE effects") and photospheric inhomogeneities ("granulation effects").

Results. We recommend use of a solar nitrogen abundance of $A(\mathrm{~N})=7.86 \pm 0.12^{\star}$, whose error bar reflects the line-to-line scatter. Conclusions. The solar metallicity implied by the CO${ }^{5}$ BOLD-based nitrogen and oxygen abundances is in the range $0.0145 \leq Z \leq$ 0.0167 . This result is a step towards reconciling photospheric abundances with helioseismic constraints on $Z$. Our most suitable estimates are $Z=0.0156$ and $Z / X=0.0213$.
\end{abstract}

Key words. Sun: abundances - stars: abundances - hydrodynamics - line: formation

\section{Introduction}

The knowledge of the precise nitrogen abundance in the solar photosphere is important because $\mathrm{N}$ accounts for about $6 \%$ (by mass) of the solar metallicity, and hence is one of the most important contributors to $Z$ after $\mathrm{O}(53 \%), \mathrm{C}(23 \%)$, and $\mathrm{Ne}(14 \%)$. Amongst others, the value of $Z$ has a crucial impact on stellar structure calculations. The abundance of $\mathrm{C}, \mathrm{N}$, and $\mathrm{O}$ in the solar system can be determined only from the solar spectrum. Meteoritic samples are un-representative, because $\mathrm{C}, \mathrm{N}$, and $\mathrm{O}$ are volatile elements. With condensation temperatures of only a few hundred Kelvin, they condense only partially so that their abundances in pristine meteorites (e.g., carbonaceous chondrites) do not provide valid estimates of the average solar system composition. The main molecule formed by $\mathrm{C}$ and $\mathrm{O}$, carbon monoxide $(\mathrm{CO})$, is also extremely stable. As a consequence, all $\mathrm{C}$ will be locked into $\mathrm{CO}$ if the original material is O-rich, and will be unable to form solid compounds. Only the remaining $\mathrm{C}$

$\star A(\mathrm{~N}) \equiv \log n(\mathrm{~N})-\log n(\mathrm{H})+12$. may condense into grains, and in the resulting meteoritic sample carbon will be underrepresented. The reverse is true if the environment is C-rich: all oxygen remains in the gas phase and the remaining $\mathrm{C}$ may form grains. Therefore, the global elemental $\mathrm{C} / \mathrm{O}$ ratio cannot be inferred from meteorites; although, isotopic ratios can be measured with high accuracy. Similar problems affect nitrogen in an even more severe manner. As a consequence, the meteoritic $\mathrm{C}, \mathrm{N}$, and $\mathrm{O}$ abundances are lower than the solar photospheric ones by large factors: $2-3$ for oxygen, about 10 for carbon, and more than 40 for nitrogen (see e.g., Palme \& Jones 2003). Analyses of observations of the solar corona also do not provide reliable abundance measurements of these elements. Several processes affect the solar corona composition, partially changing the abundance of some elements (Anders \& Grevesse 1989; Grevesse \& Sauval 2000). The solar nitrogen abundance can be derived reliably only from the photospheric spectrum.

Besides its importance in determining the solar metallicity, nitrogen is an interesting element from a nucleosynthetic point of view. Its main production channel is the $\mathrm{CNO}$ cycle, in which it is produced at the expense of $\mathrm{C}$ and $\mathrm{O}$. The main astrophysical 
$\mathrm{N}$ production sites remain unclear; AGB stars are good candidates, in addition to rotating massive stars. Nitrogen is measured in many different objects: hot stars, cool stars, Galactic and extragalactic H II regions, and Damped Ly $\alpha$ systems at high redshift. For all of these studies, a good solar reference value is of fundamental importance.

The measurements of heavy element abundances inferred from photospheric solar spectra should agree with results from helioseismology. Helioseismology can provide a measurement of the solar metallicity in essentially three ways: i) from the depth of the convection zone: this depends sensitively on the opacity at the base of the convection zone, which in turn is a function of the abundance of heavy elements (Basu \& Antia 1997); ii) using information from the core: the small-frequency spacings of low-degree modes and their separation ratios are sensitive to the mean molecular weight in the core, which can be related to the metallicity of the outer layers (Basu et al. 2007); iii) from the sound speed gradient in the ionisation zone: the depth profile of this quantity can be inferred from helioseismic inversions, and comparison with the results of theoretical solar structure models of different metallicity allows us to place tight constraints on $Z$ (Antia \& Basu 2006). A comprehensive review on helioseismology and solar abundances is given by Basu \& Antia (2008) which the reader is referred to for further details. It is remarkable that all helioseismic methods provide results that are fairly consistent with each other, which infer a higher metallicity than currently deduced from the analysis of the photospheric spectrum.

In this paper, we determine the photospheric nitrogen abundance by using data for atomic lines and the latest CO ${ }^{5}$ BOLD solar model. One of our aims is to combine this result with our other solar abundance determinations to obtain a measure of the solar $Z$ based on the CO ${ }^{5}$ BOLD solar model, and to compare this with the $Z$ values inferred by helioseismology.

\section{Nitrogen abundance indicators}

\subsection{Forbidden lines}

The strongest forbidden [ $\mathrm{N} \mathrm{I}]$ lines within the ground configuration are close to $1040.0 \mathrm{~nm}$ and belong to the multiplet ${ }^{2} \mathrm{D}^{0}-{ }^{2} \mathrm{P}^{0}$. Houziaux (1961) claimed to detect these lines in the solar spectrum, although studies by Lambert \& Swings (1967) and Swensson (1967) could not confirm these detections. Swensson (1967) also demonstrated convincingly that the predicted equivalent widths are too weak to be detectable in the Jungfraujoch Atlas.

\subsection{Permitted lines}

The permitted Ni lines of low excitation potential occur at UV wavelengths, mostly in the $80-120 \mathrm{~nm}$ range. The atomic $\mathrm{N}$ I lines measurable in the solar spectrum are found in the visual and near IR range, and all have a high excitation potential $\left(E_{\text {low }}>10.3 \mathrm{eV}\right)$, implying that these lines are formed in the deep layers of the photosphere. They are therefore hardly affected by departures from LTE $(\triangle A(\mathrm{~N}) \sim-0.05 \mathrm{dex}$, Rentzsch-Holm 1996). In the classical analysis that is based on $1 \mathrm{D}$ model atmospheres, these lines have the disadvantage of being very sensitive to the temperature structure of the deep layers, which, for theoretical models, depends on the treatment of convection and, most significantly, on the choice of the mixing-length parameter (see Fig. 1). In principle, these difficulties are overcome by

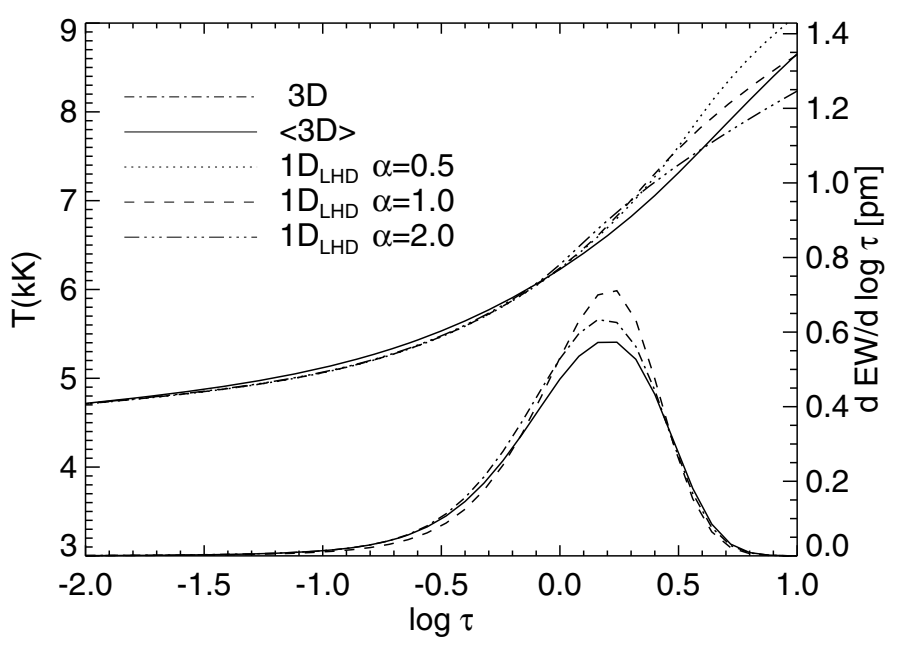

Fig. 1. The temporal and horizontal average of the temperature profile of the $3 \mathrm{D}$ model (solid line) and the temperature profile of $1 \mathrm{D}_{\mathrm{LHD}}$ models of three values of $\alpha_{\mathrm{MLT}}$, are shown as a function of (Rosseland) optical depth. In addition, equivalent width contribution functions (lower, roughly Gaussian-shaped curves) for the $746.8 \mathrm{~nm}$ line at disc-centre are plotted as a function of monochromatic continuum optical depth.

modern 3D hydrodynamical simulations, which provide a physically consistent description of convection from first principles.

\subsection{Molecular lines}

Grevesse et al. (1990) illustrated that atomic and molecular lines react in opposite directions to temperature perturbations, so that the agreement between the atomic and molecular lines provides support for the choice of solar model. In their molecular analysis, they and other authors have relied on the use of $\mathrm{NH}$ and $\mathrm{CN}$ molecular lines. In this paper, we derive the solar $\mathrm{N}$ abundance from only the atomic lines and defer the study of molecular lines to the future. On the one hand the abundance of carbon has not yet been estimated with a $\mathrm{CO}^{5} \mathrm{BOLD}$ model, and on the other hand, synthesising the NH A-X band, used e.g. by Lambert (1978) in the solar spectrum, would require accounting for a large number of lines, both $\mathrm{NH}$ lines and blending atomic lines, and the state-of the art version of Linfor3D is unable to handle hundreds of lines. Potential deviations from LTE for the molecular species is another issue that hampers the use of molecular lines for accurate abundance analysis. For these reasons, we postpone the study of molecular lines harbouring $\mathrm{N}$.

\subsection{Line selection}

The first selection of lines was completed by extracting all the Ni lines from the NIST Atomic Spectra Database (NIST ASD Ralchenko 2005) in the range 400-1200 nm. These lines were then checked by visual inspection in the solar atlases of Delbouille et al. (1973) and Delbouille et al. (1981); the identifications were achieved by using the Utrecht spectrum identifications (Moore et al. 1966) for the 400-877 nm range, and those by Swensson et al. (1970) for the 900-1200 nm range. According to the NIST ASD, no N I line is present in the $877-900 \mathrm{~nm}$ range, which is not covered by these identification lists. We also performed a check of the relative line intensities with the Moore Multiplet Table (Moore 1945). The strongest and least blended lines are given in Table 1 with the $\log g f$ values, and their qualities taken from the NIST ASD. 
Table 1. Selection of permitted N I lines in the visual and near IR spectral range.

\begin{tabular}{rcclrr}
\hline \hline$\lambda(\mathrm{nm})$ & $E_{\text {low }}(\mathrm{eV})$ & $\log g f$ & $Q$ & $n_{\text {low }}$ & $n_{\text {up }}$ \\
\hline 744.229 & 10.330 & -0.385 & $\mathrm{~B}+$ & 4 & 10 \\
746.831 & 10.336 & -0.190 & $\mathrm{~B}+$ & 4 & 10 \\
821.634 & 10.336 & +0.132 & $\mathrm{~B}+$ & 4 & 9 \\
822.314 & 10.330 & -0.271 & $\mathrm{~B}+$ & 4 & 9 \\
868.340 & 10.330 & +0.087 & $\mathrm{~B}+$ & 4 & 8 \\
871.883 & 10.336 & -0.336 & $\mathrm{~B}+$ & 4 & 8 \\
1010.513 & 11.750 & +0.219 & $\mathrm{~B}+$ & 8 & 17 \\
1011.248 & 11.758 & +0.607 & $\mathrm{~B}+$ & 8 & 17 \\
1011.464 & 11.764 & +0.768 & $\mathrm{~B}+$ & 8 & 17 \\
1050.700 & 11.840 & +0.094 & $\mathrm{~B}$ & 9 & 20 \\
1052.058 & 11.840 & +0.010 & $\mathrm{~B}$ & 9 & 20 \\
1053.957 & 11.844 & +0.503 & $\mathrm{~B}$ & 9 & 20 \\
\hline
\end{tabular}

Notes: error of $\log g f \leq 0.03 \operatorname{dex}(Q=\mathrm{B}+), \leq 0.08 \operatorname{dex}(Q=\mathrm{B})$; $n_{\text {low }}$ and $n_{\text {up }}$ identify the level of the nitrogen model atom used for the NLTE computations (cf. Fig. 3).

\section{Models and line-formation codes}

\subsection{D hydrodynamical model atmosphere}

Our analysis is based mainly on a 3-dimensional radiationhydrodynamics simulation (hereafter 3D model) computed with the CO5 BOLD code (Freytag et al. 2002, 2003; Wedemeyer et al. 2004). Some basic information about the setup of this numerical simulation can be found in Caffau et al. (2008), who used the same model to determine the solar oxygen abundance. A full description of the model, including a critical review of its performance in reproducing various observational constraints, will be given in Ludwig et al. (2009).

\section{2. $1 D$ reference models}

For comparison, we also used several 1D solar models:

1. The semi-empirical Holweger-Müller model (Holweger 1967; Holweger \& Müller 1974, hereafter HM); when necessary, this was placed on a mass column-density scale, using the opacity used to produce the CO5 BOLD model.

2. A 1D model computed with the LHD code, which uses the same microphysics as CO ${ }^{5}$ BOLD and treats convection with the mixing-length approximation, adopting $\alpha_{\mathrm{MLT}}=1.0$ (see Caffau \& Ludwig 2007, for further details), and two further $1 \mathrm{D}_{\mathrm{LHD}}$ models with $\alpha_{\mathrm{MLT}}$ of 0.5 and 2.0, to investigate the dependence of the abundance on $\alpha_{\text {MLT }}$.

3. The ATLAS9 solar model with the abundances of Asplund et al. (2005) as computed by Castelli ${ }^{1}$.

4. A $1 \mathrm{D}$ model obtained by temporal and horizontal averaging over surfaces of equal (Rosseland) optical depth and over all snapshots. We refer to this averaged model as $\langle 3 \mathrm{D}\rangle$.

\subsection{Line-formation calculations}

The 3D spectrum synthesis computations are all performed with Linfor $3 D^{2}$, which can also compute line formation using as input different type of 1D models. For comparison in the case of

\footnotetext{
1 http://wwwuser.oats.inaf.it/castelli/sun/ ap00t5777g44377k1asp. dat

2 http://www.aip.de/ mst/Linfor3D/linfor_3D_manual . pdf
}

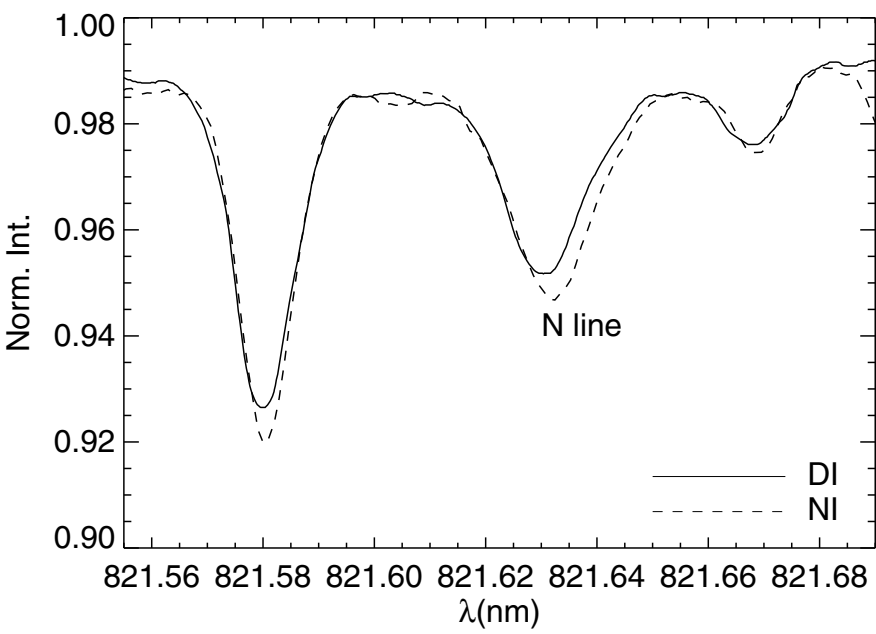

Fig. 2. Observed profile of the NI line at $\lambda 821.6 \mathrm{~nm}$ as extracted from the Delbouille (solid) and Neckel (dashed) solar disc-centre intensity spectral atlases. The reason for the considerable differences in unknown.

1D models, we also used the SYNTHE code (Kurucz 1993, 2005b) in its Linux version (Sbordone et al. 2004; Sbordone 2005) in calculating synthetic spectra. The advantage of SYNTHE over Linfor3D is that it can easily treat hundreds of thousands of lines, while the present version of Linfor3D is limited to a few tens of lines.

\section{Observational data}

Investigations of solar abundances found in the literature usually provide detailed discussions about both the accuracy of the adopted model atmospheres and the related uncertainties in the derived abundances. However, the accuracy of the observational data is often not taken into consideration, since, typically, solar abundance determinations rely on a single observed spectrum.

\subsection{Differences between high quality solar spectra}

In our study of the solar oxygen abundance (Caffau et al. 2008), we noted that different high quality solar spectra exhibit differences that were larger than expected from their high $\mathrm{S} / \mathrm{N}$ ratios. To investigate the problem related to the differences between the solar atlases in the case of nitrogen, we checked four selected lines. From this non-exhaustive analysis, we conclude, as we did for other elements, that for some, but not all, lines, the solar atlases disagree. This is the case for one of four nitrogen lines that we considered as illustrated in Fig. 2. The presence of telluric absorption might explain this difference. However, by analysing spectra of rapidly rotating hot stars, there is no evidence of any telluric contamination, but the day-time sky can exhibit absorption that is undetectable at night-time. An indisputable reason for the differences still needs to be identified. We strongly believe that the astronomical community needs a new high-quality solar atlas.

\subsection{Comparison of different EW measurements}

Inspection of the literature often reveals that even the measurement of equivalent widths ( $E W \mathrm{~s}$ ) from one and the same observed spectrum is less straightforward than what could be expected. Different authors use different approaches and measure different equivalent widths. A recent example is the comparison 
of our EWs for oxygen (Caffau et al. 2008) with those of Asplund et al. (2004). A long-running debate about the solar iron abundance has in part been driven by differences in the $E W \mathrm{~s}$ (see for example Holweger et al. 1995, and references therein). For the nitrogen, the $E W$ measurements differ also between different works. Comparing the measurements of the $E W$ s of two authoritative works such as Grevesse et al. (1990) and Biémont et al. (1990), we conclude that there is good agreement for our selected lines of nitrogen. The authors measure similar values without any systematic differences being evident.

\subsection{Data used in this study}

In our analysis, we used the $E W$ s of Grevesse et al. (1990) and Biémont et al. (1990), which are in excellent agreement.

For a few selected lines, we derived in addition the nitrogen abundance from detailed line profile fitting (see Fig. 5). For this purpose, we used the two centre-disc intensity atlases (the "Delbouille" atlas, i.e., Delbouille et al. 1973; Delbouille et al. 1981; and the "Neckel" intensity atlas; Neckel \& Labs 1984), as well as the two solar flux atlases (the "Kurucz" solar flux atlas Kurucz 2005a; and the "Neckel" solar flux atlas Neckel \& Labs 1984). Further details about these atlases can be found in the aforementioned references and Caffau et al. (2008).

\section{NLTE computations}

The nitrogen lines considered in our analysis are weak and form deep inside the solar photosphere (see Fig. 1, showing the $E W$ contribution function for one of the nitrogen lines). From these facts, we do not expect that these lines are very sensitive to departures from Local Thermodynamic Equilibrium (LTE). In her analysis of the statistical equilibrium of nitrogen in the Sun, Rentzsch-Holm (1996) inferred that the deviations from LTE are small and negative, on average about -0.05 dex. In this paper, we confirm this result.

\subsection{D NLTE corrections}

Since the nitrogen lines form at the same photospheric depth range in both $3 \mathrm{D}$ and $1 \mathrm{D}\left(\langle 3 \mathrm{D}\rangle, 1 \mathrm{D}_{\mathrm{LHD}}\right)$ models (see contribution functions in Fig. 1), this infers that 3D granulation effects do not play a fundamental role. Since the contribution functions for $3 \mathrm{D}$ and 1D models are similar, it follows that the computed $E W \mathrm{~s}$ are also similar, implying that the $3 \mathrm{D}$ corrections are small. Without a code capable of solving the full 3D-NLTE problem for nitrogen, we resort to 1D-NLTE calculations.

We computed the departures from LTE for the $\langle 3 \mathrm{D}\rangle$ and HM models, using the Kiel code (Steenbock \& Holweger 1984), with the model atom of Rentzsch-Holm (1996). The Kiel code uses a generalisation of the Drawin (1969) formalism to take into account excitation and ionisation of the nitrogen atoms by inelastic collisions with neutral hydrogen atoms. A scaling factor, $S_{\mathrm{H}}$, permits to reduce the efficiency of these collisions $\left(S_{\mathrm{H}}<1\right)$, to switch them off $\left(S_{\mathrm{H}}=0\right)$, or to consider them "in toto" $\left(S_{\mathrm{H}}=1\right)$. Consistent with the Kiel group and in line with Rentzsch-Holm (1996), we favour $S_{\mathrm{H}}=1 / 3$, even though we computed NLTE corrections in addition for $S_{\mathrm{H}}=0$ and $S_{\mathrm{H}}=1$. In Fig. 3, the departure coefficients of the atomic levels relevant to the selected lines (see Table 1) are shown for the $\langle 3 \mathrm{D}\rangle$ model.

The NLTE corrections obtained from the $\langle 3 \mathrm{D}\rangle$ and the HM model are given in Table 4. They are added to the abundances obtained from the LTE analysis based on the 3D and

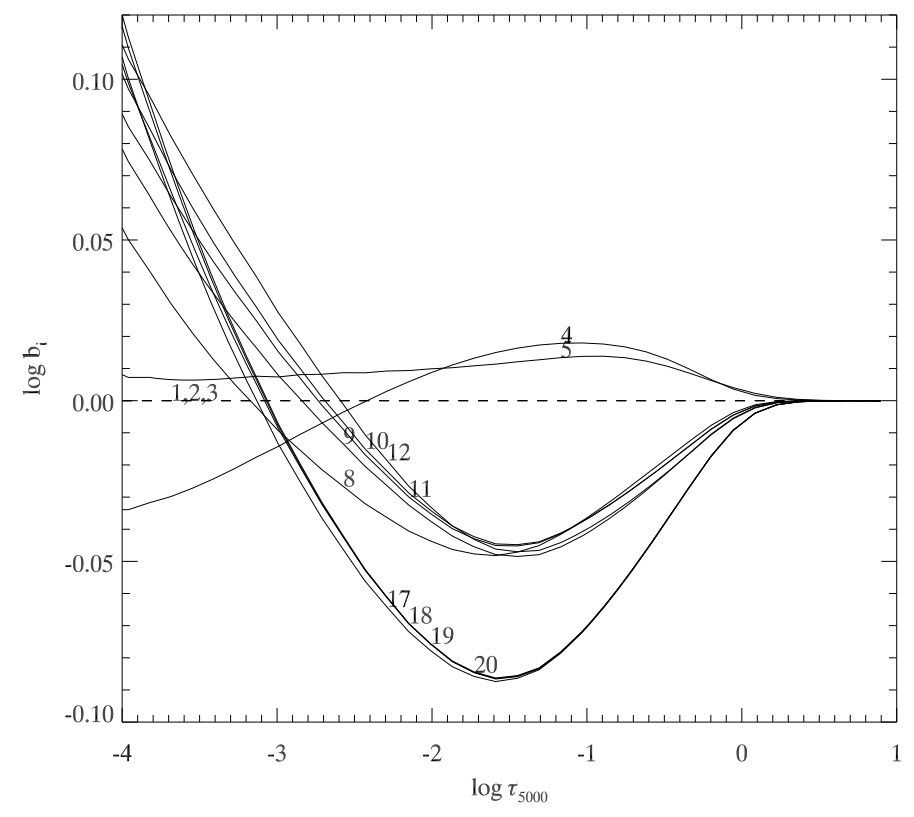

Fig. 3. Departure coefficients $\log b_{i}=\log \left(n_{\mathrm{NLTE}} / n_{\mathrm{LTE}}\right)$ of selected levels of neutral nitrogen as a function of optical depth $\log \tau_{5000}$. For each line involved in our abundance analysis, the identification of the upper and lower level is listed in Table 1 . The shown $b_{i}$ refer to the $\langle 3 \mathrm{D}\rangle$ model; similar results are obtained for the HM model.

the HM model, respectively, to obtain the final NLTE nitrogen abundances.

\subsection{Influence of horizontal temperature fluctuations}

Using the $\langle 3 \mathrm{D}\rangle$ model to compute departures from LTE is an extreme case in which all horizontal temperature fluctuations are neglected. To estimate how this approximation affects departures from LTE, we produced horizontal and time-averaged models by grouping columns into twelve bins according to their emergent continuum intensity in the vertical direction. A similar procedure was used by Aufdenberg et al. (2005) to estimate the effects of horizontal, temperature inhomogeneities.

These twelve components represent the different horizontal temperature structures associated with the presence of granules and intergranular lanes. Each component has a weight that depends on its surface area fraction. The cool downdrafts are associated with the groups of lowest intensity, and the warm upflows with those of the highest intensity. We computed NLTE corrections using each of these components, which were treated as standard plane-parallel model atmospheres (1.5D approximation). The components allowed us to study the variations in the NLTE corrections as a function of the temperature structure of the flow where the line is formed. The results, for a selection of lines, are shown in Fig. 4.

Lines with excitation energies higher than $11 \mathrm{eV}$ exhibit little differences in departures from LTE for the twelve groupaveraged models. For the lines with lower excitation energies $\left(E_{\text {low }} \approx 10.3 \mathrm{eV}\right)$, the groups corresponding to the cool downdrafts (groups 1 to 6 ) have a correction that is distinctly larger (in absolute value) than for the groups corresponding to the warm upflows (groups 7 to 12). We consider the variation in the NLTE corrections of the various components, relative to the result of the $\langle 3 \mathrm{D}\rangle$ model, as an indication of the uncertainty associated with the $1 \mathrm{D}$ approximation. 
Table 2. $A(\mathrm{~N})_{\mathrm{LTE}}$ from selected $\mathrm{N}$ l lines with $E W \mathrm{~s}$ from the literature using Linfor3D.

\begin{tabular}{|c|c|c|c|c|c|c|c|c|c|c|c|c|}
\hline \multirow{2}{*}{$\begin{array}{r}\lambda \\
(\mathrm{nm}) \\
(1)\end{array}$} & \multicolumn{2}{|c|}{$\begin{array}{c}E W \\
(\mathrm{pm})\end{array}$} & \multicolumn{2}{|c|}{$\overline{\bar{A} A(\mathrm{~N}) 3 \mathrm{D}}$} & \multicolumn{2}{|c|}{$\overline{A A(\mathrm{~N})\langle 3 \mathrm{D}\rangle}$} & \multicolumn{2}{|c|}{ 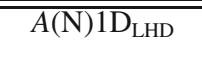 } & \multicolumn{2}{|c|}{ 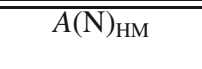 } & \multirow{2}{*}{ 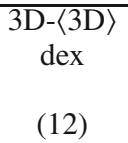 } & \multirow{2}{*}{$\begin{array}{c}\text { 3D-1D } \\
\text { dex } \\
(13)\end{array}$} \\
\hline & $\begin{array}{l}\mathrm{G} \\
(2)\end{array}$ & $\begin{array}{c}\text { B } \\
\text { (3) }\end{array}$ & $\begin{array}{l}\text { G } \\
\text { (4) }\end{array}$ & $\begin{array}{c}\mathrm{B} \\
\text { (5) }\end{array}$ & $\begin{array}{c}G \\
\text { (6) }\end{array}$ & $\begin{array}{l}\text { B } \\
\text { (7) }\end{array}$ & $\begin{array}{c}\mathrm{G} \\
(8)\end{array}$ & $\begin{array}{c}\text { B } \\
\text { (9) }\end{array}$ & $\begin{array}{c}\mathrm{G} \\
\text { (10) }\end{array}$ & $\begin{array}{c}\mathrm{B} \\
(11)\end{array}$ & & \\
\hline 744.2 & 0.26 & 0.27 & 7.808 & 7.826 & 7.847 & 7.865 & 7.810 & 7.828 & 7.911 & 7.928 & -0.039 & -0.002 \\
\hline 746.8 & 0.52 & 0.49 & 7.961 & 7.931 & 7.994 & 7.964 & 7.954 & 7.924 & 8.057 & 8.027 & -0.033 & +0.008 \\
\hline 821.6 & 0.86 & 0.87 & 7.854 & 7.860 & 7.892 & 7.899 & 7.847 & 7.853 & 7.957 & 7.963 & -0.039 & +0.007 \\
\hline 822.3 & 0.24 & & 7.593 & & 7.648 & & 7.611 & & 7.718 & & -0.055 & -0.018 \\
\hline 868.3 & 0.78 & 0.81 & 7.828 & 7.849 & 7.865 & 7.885 & 7.821 & 7.841 & 7.929 & 7.949 & -0.037 & +0.007 \\
\hline 871.8 & 0.42 & 0.43 & 7.927 & 7.939 & 7.971 & 7.983 & 7.933 & 7.944 & 8.036 & 8.047 & -0.044 & -0.006 \\
\hline 1010.5 & 0.18 & & 7.956 & & 8.022 & & 7.976 & & 8.098 & & -0.066 & -0.020 \\
\hline 1011.2 & 0.35 & 0.36 & 7.897 & 7.912 & 7.958 & 7.971 & 7.908 & 7.922 & 8.033 & 8.046 & -0.060 & -0.011 \\
\hline 1011.4 & 0.55 & 0.54 & 7.976 & 7.966 & 8.028 & 8.019 & 7.976 & 7.967 & 8.101 & 8.092 & -0.053 & -0.001 \\
\hline 1050.7 & 0.14 & & 8.002 & & 8.066 & & 8.022 & & 8.143 & & -0.064 & -0.020 \\
\hline 1052.0 & 0.08 & & 7.829 & & 7.896 & & 7.853 & & 7.975 & & -0.067 & -0.024 \\
\hline 1053.9 & 0.32 & & 7.989 & & 8.046 & & 7.999 & & 8.121 & & -0.057 & -0.010 \\
\hline Average & & & 7.885 & 7.890 & 7.936 & 7.941 & 7.892 & 7.897 & 8.007 & 8.007 & -0.051 & -0.008 \\
\hline
\end{tabular}

Notes: columns with G are from Grevesse et al. (1990), with B are from Biémont et al. (1990). Column (1) is the wavelength; Cols. (2), (3) the $E W$ s; Cols. (4)-(11) $A(\mathrm{~N})$ from 3D, $\langle 3 \mathrm{D}\rangle, 1 \mathrm{D}_{\mathrm{LHD}}$, and HM model; Cols. (12), (13) two different 3D corrections.

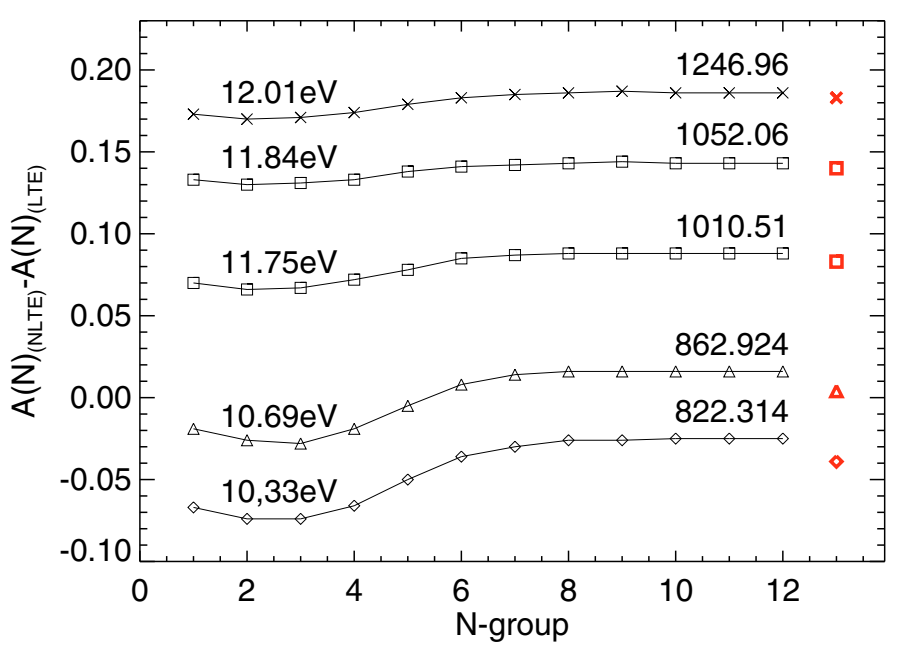

Fig. 4. For five representative lines the 1D-NLTE corrections from the twelve group-averaged models, ordered according to increasing continuum intensity (radiation temperature) from left to right, are shown together with the result for the global $\langle 3 \mathrm{D}\rangle$ model (rightmost, bold symbol). The lowest curve is plotted at the true ordinate level, while each of the others curves is shifted up by 0.05 dex with respect to the previous one for clarity.

The variations in the NLTE-corrections are small, even for the lower excitation lines, for which the difference between the extreme groups is less than 0.05 dex. We therefore expect that the results we obtained using the $\langle 3 \mathrm{D}\rangle$ model will not disagree by more than 0.03 dex with the results of a full 3D-NLTE computation.

\section{Nitrogen abundance determinations}

\subsection{LTE abundance from selected lines}

The following abundance analysis is based on a subsample of lines given in Table 1 . We determined individual LTE nitrogen abundances, $A(\mathrm{~N})_{\mathrm{LTE}}$ using the $3 \mathrm{D},\langle 3 \mathrm{D}\rangle, 1 \mathrm{D}_{\mathrm{LHD}}$, and HM model, respectively, and the equivalent width measurements by Grevesse et al. (1990) and Biémont et al. (1990) where available. The results are given in Table 2. In this table and in the others following, we indicate the abundances to three decimal places, following the prescription of Bevington \& Robinson (2003) of retaining one more significant digit than dictated by the error. For nitrogen lines, which are formed in the deep photosphere, the $3 \mathrm{D}$ correction, defined as $A(\mathrm{~N})_{3 \mathrm{D}}-A(\mathrm{~N})_{1 \mathrm{D}_{\mathrm{LHD}}}$, Col. (13), is small, in the range $-0.024-0.008$ dex. The "granulation abundance correction", $A(\mathrm{~N})_{3 \mathrm{D}}-A(\mathrm{~N})_{\langle 3 \mathrm{D}\rangle}$, (indicated in Col. (12) of Table 2), which measures the influence of horizontal fluctuations, is somewhat larger, and negative for all the lines. We reported similar behaviour for the O I $615.8 \mathrm{~nm}$ line in previous work (see Caffau et al. 2008).

\subsection{Line-profile fitting}

We selected four clean and not too weak lines from the set of lines in Table 1 (746.8 nm, $821.6 \mathrm{~nm}, 868.3 \mathrm{~nm}$, and $1011.4 \mathrm{~nm})$ for which we fitted the line profile for all the four observed atlases (two fluxes and two centre-disc intensities), using 1D models with SYNTHE as a line formation code. We discarded the line at $1011.4 \mathrm{~nm}$ for which we could not obtain a reliable fit; the abundance that we derived from this line was also too high to be consistent with those found for other lines, although we note that the four different spectra consistently indicated the same (high) abundance. The line list used in the profile fitting of this line appears to be incomplete. Nevertheless, we decide to retain this line in the abundance determination inferred from the $E W \mathrm{~s}$, because there it is consistent with the other values, indicating that the line decomposition method used to determine $E W$ is reliable.

The $A(\mathrm{~N})$ derived from the line at $821.6 \mathrm{~nm}$ in the four different spectra exhibits a scatter of $0.050 \mathrm{dex}$, when considering the $\langle 3 \mathrm{D}\rangle$-NLTE abundance, which is considerably larger than the scatter found for the other two lines. The difference between the $A(\mathrm{~N})$ derived from the two disc-integrated spectra is about 0.06 dex, while the $A(\mathrm{~N})$ from the two centre-disc spectra differ by about 0.13 dex. Neither the two disc-integrated spectra nor the two centre-disc spectra do agree (see Fig. 2). The difference in $E W$ is of the order of $15 \%$. This is much larger than expected from the high $\mathrm{S} / \mathrm{N}$ ratio of the spectra.

The line fitting results are given in Table 3. An example of the excellent agreement achieved in the line fitting using the HM model, is shown in Fig. 5. 
Table 3. $A(\mathrm{~N})$ of N I from line fitting using 1D models with SYNTHE.

\begin{tabular}{cccccc}
\hline \hline $\begin{array}{c}\text { Observed } \\
\text { spectrum }\end{array}$ & $\begin{array}{c}\lambda \\
(\mathrm{nm})\end{array}$ & $\mathrm{HM}$ & $\begin{array}{c}A(\mathrm{~N})_{\text {LTE }} \\
\text { ATLAS9 }\end{array}$ & $\langle 3 \mathrm{D}\rangle$ & $\begin{array}{c}A(\mathrm{~N})_{\text {NLTE }} \\
\langle 3 \mathrm{D}\rangle\end{array}$ \\
\hline $\mathrm{KF}$ & 746.8 & 7.861 & 7.824 & 7.809 & 7.750 \\
$\mathrm{NF}$ & 746.8 & 7.874 & 7.837 & 7.827 & 7.768 \\
$\mathrm{NI}$ & 746.8 & 7.890 & 7.834 & 7.823 & 7.785 \\
$\mathrm{DI}$ & 746.8 & 7.908 & 7.849 & 7.840 & 7.802 \\
\hline $\mathrm{KF}$ & 821.6 & 7.847 & 7.811 & 7.805 & 7.730 \\
$\mathrm{NF}$ & 821.6 & 7.918 & 7.870 & 7.867 & 7.792 \\
$\mathrm{NI}$ & 821.6 & 7.944 & 7.879 & 7.873 & 7.821 \\
$\mathrm{DI}$ & 821.6 & 7.808 & 7.752 & 7.746 & 7.694 \\
\hline $\mathrm{KF}$ & 868.3 & 7.910 & 7.889 & 7.857 & 7.783 \\
$\mathrm{NF}$ & 868.3 & 7.906 & 7.877 & 7.857 & 7.783 \\
$\mathrm{NI}$ & 868.3 & 7.933 & 7.878 & 7.875 & 7.831 \\
$\mathrm{DI}$ & 868.3 & 7.932 & 7.886 & 7.882 & 7.838 \\
\hline
\end{tabular}

Notes: Col. (1) is the observed spectrum identification, KF: Kurucz Flux, NF: Neckel Flux, NI: Neckel Intensity, and DI: Delbouille Intensity; Col. (2) is the wavelength; Cols. (3)-(5) the $A(\mathrm{~N})_{\mathrm{LTE}}$ from line fitting with HM, ATLAS9, and $\langle 3 \mathrm{D}\rangle$ model; Col. (6) is the $A(\mathrm{~N})_{\mathrm{NLTE}}$ for the $\langle 3 \mathrm{D}\rangle$ model, obtained by adding the NLTE-correction computed with $S_{\mathrm{H}}=1 / 3$ to the abundances given in Col. (5).

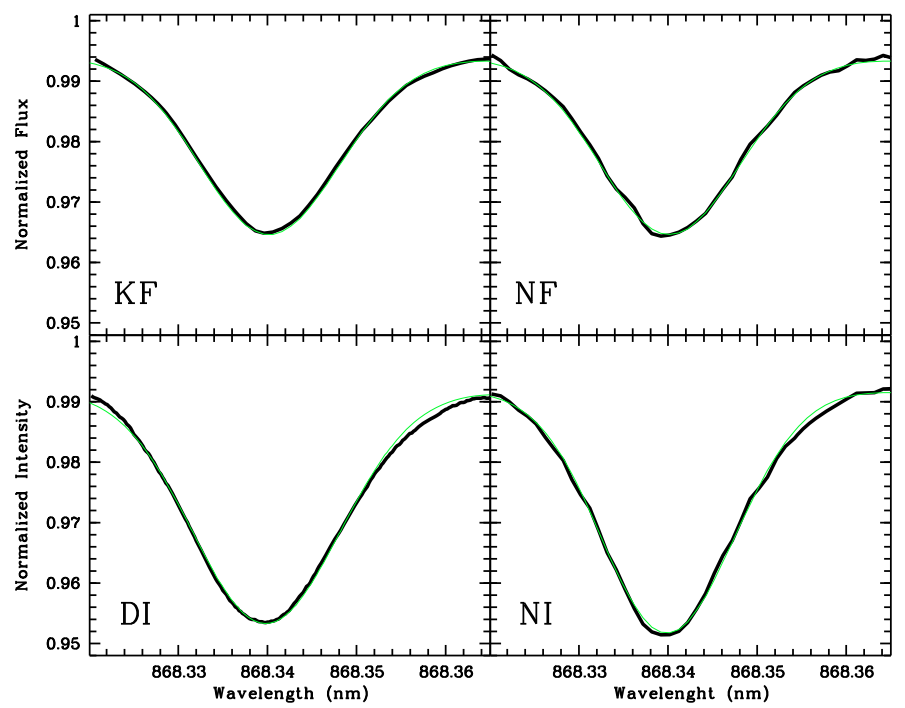

Fig. 5. Results of the line profile fits for the $868.3 \mathrm{~nm}$ line. The synthetic spectra computed with SYNTHE and using the Holweger-Müller model (thin lines) are superimposed on the observed spectra from the four solar atlases $(\mathrm{KF}=$ Kurucz flux, NF $=$ Neckel Flux, DI = Delbouille Intensity, $\mathrm{NI}=$ Neckel Intensity; thick lines).

The differences between the nitrogen abundance from both the fitting procedure and by matching the measured $E W \mathrm{~s}$ can be explained by the influence of blending components, which are accounted for in different ways in the line-fitting approach and in the $E W$ method, respectively. The different codes used may also be responsible for part of the difference.

Since the majority of the nitrogen lines are blended, weak, or both, the line-fitting approach is, in principle, more appropriate. However, line fitting with 3D spectra can become a tedious task, and we therefore prefer to rely on equivalent width measurements. We believe that we cannot achieve superior measurement of the $E W$ s than the careful works by Grevesse et al. (1990) and Biémont et al. (1990). In principle, we could also measure the $E W \mathrm{~s}$ in data from the other atlases, but our above line fitting exercise implies that this would introduce an additional scatter of the order of at most approximately $0.03 \mathrm{dex}$, due to the

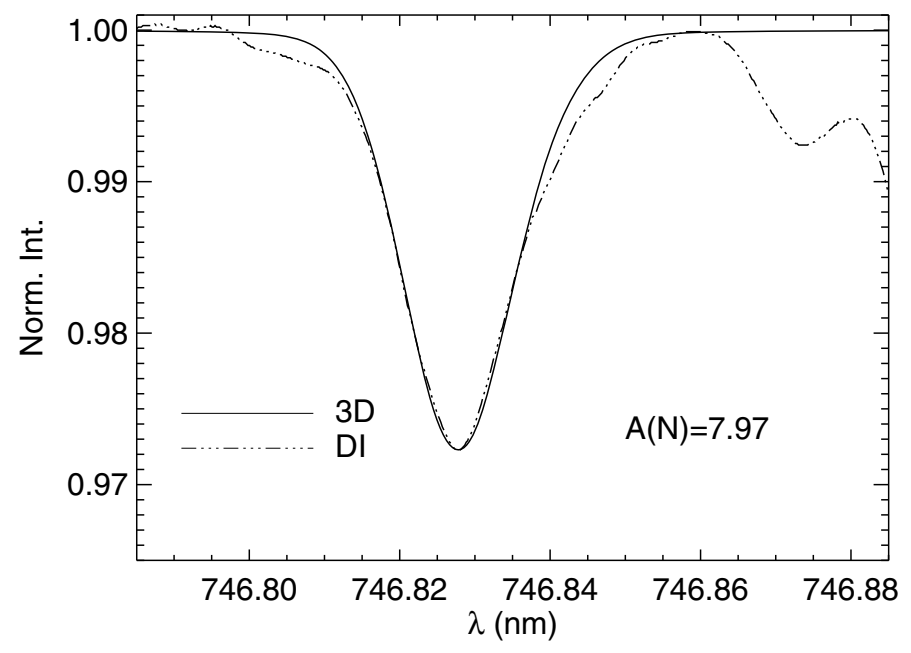

Fig. 6. The unblended 3D profile of N I $746.8 \mathrm{~nm}$ is compared to the observed solar centre-disc profile of the Neckel Intensity atlas.

differences between atlases. As we shall see, this is much less than the scatter derived from the different lines. Our final choice is therefore to derive $A(\mathrm{~N})$ from the $E W \mathrm{~s}$ from Grevesse et al. (1990) and Biémont et al. (1990), using both 3D and 1D models.

As a consistency check, we also compared the 3D profiles with the observed spectra, as shown for one example in Fig. 6. The agreement is in general good, given that the blending line visible in the observed spectrum were not considered in the synthetic 3D profile.

\subsection{D-NLTE abundance}

Table 4 provides the NLTE corrections for both the $\langle 3 \mathrm{D}\rangle$ and the HM models. Our final 3D-NLTE nitrogen abundance is obtained by averaging the 12 individual 3D-NLTE abundances (Col. (2) + Col. (4)), with equal weight. The individual as well as the averaged NLTE-corrections are given in Cols. (4)-(6), respectively. The result is:

$A(\mathrm{~N})=7.85 \pm 0.12$ for $S_{\mathrm{H}}=0$
$A(\mathrm{~N})=7.86 \pm 0.12$ for $S_{\mathrm{H}}=1 / 3$
$A(\mathrm{~N})=7.87 \pm 0.12$ for $S_{\mathrm{H}}=1$.

With the EWs from Biémont et al. (1990), $A(\mathrm{~N})$ remains the same while the scatter is reduced to $0.06 \mathrm{dex}$, since the scatter is insensitive to the choice of $S_{\mathrm{H}}$ due to these lines being formed at similar depths in the solar photosphere and having similar, and, in any case, small, NLTE corrections.

\section{Discussion}

\section{1. $3 D$ effects and NLTE-corrections}

A decisive point in favour of using 3D hydrodynamical models (or a semi-empirical model) is that there is no need to invoke mixing-length theory. All the nitrogen lines used in our abundance determination arise from transitions between levels of high excitation energy, so that they are formed deep in the solar photosphere, in the range $-1<\log \tau<+1$, and hence are sensitive to the choice of the mixing-length parameter in classical 1D models. A change of ${ }_{-0.5}^{+1.0}$ in the value of the mixinglength parameter of 1.0 that we adopt, produces a change in the abundance with the $1 \mathrm{D}_{\text {LHD }}$ model of on average ${ }_{+0.05}^{-0.09}$ dex. The "granulation abundance correction", $A(\mathrm{~N})_{3 \mathrm{D}}-A(\mathrm{~N})_{\langle 3 \mathrm{D}\rangle}$, is 
Table 4. $A(\mathrm{~N})_{\mathrm{LTE}}$ and $\Delta A(\mathrm{~N})_{\mathrm{NLTE}}$ from selected N I lines with disc-centre $E W$ s from the literature obtained with Linfor3D and Kiel code for NLTE computations.

\begin{tabular}{|c|c|c|c|c|c|c|c|c|c|c|}
\hline \multirow{4}{*}{$\begin{array}{r}\lambda(\mathrm{nm}) \\
(1)\end{array}$} & \multirow{2}{*}{\multicolumn{2}{|c|}{$\overline{A(\mathrm{~N}) 3 \mathrm{D}_{\mathrm{LTE}}}$}} & \multirow{2}{*}{\multicolumn{3}{|c|}{$\begin{array}{c}\Delta A(\mathrm{~N})_{\mathrm{NLTE}}\langle 3 \mathrm{D}\rangle \\
S_{\mathrm{H}}\end{array}$}} & \multirow{2}{*}{\multicolumn{2}{|c|}{ 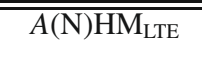 }} & \multirow{2}{*}{\multicolumn{3}{|c|}{$\begin{array}{c}\Delta A(\mathrm{~N})_{\text {NLTE }} \\
S_{\mathrm{H}}\end{array}$}} \\
\hline & & & & & & & & & & \\
\hline & G & B & $1 / 3$ & 1 & 0 & G & B & $1 / 3$ & 1 & 0 \\
\hline & (2) & (3) & (4) & (5) & (6) & (7) & (8) & (9) & (10) & (11) \\
\hline 744.2 & 7.808 & 7.826 & -0.034 & -0.026 & -0.038 & 7.911 & 7.928 & -0.038 & -0.028 & -0.042 \\
\hline 746.8 & 7.961 & 7.931 & -0.038 & -0.030 & -0.042 & 8.056 & 8.027 & -0.043 & -0.032 & -0.047 \\
\hline 821.6 & 7.854 & 7.860 & -0.052 & -0.037 & -0.064 & 7.957 & 7.963 & -0.057 & -0.041 & -0.071 \\
\hline 822.3 & 7.593 & & -0.039 & -0.028 & -0.049 & 7.718 & & -0.045 & -0.032 & -0.056 \\
\hline 868.3 & 7.828 & 7.849 & -0.047 & -0.034 & -0.061 & 7.929 & 7.949 & -0.051 & -0.036 & -0.065 \\
\hline 871.8 & 7.927 & 7.939 & -0.040 & -0.029 & -0.052 & 8.036 & 8.047 & -0.044 & -0.032 & -0.058 \\
\hline 1010.4 & 7.956 & & -0.017 & -0.012 & -0.025 & 8.098 & & -0.020 & -0.015 & -0.029 \\
\hline 1011.2 & 7.897 & 7.912 & -0.019 & -0.014 & -0.028 & 8.033 & 8.046 & -0.023 & -0.016 & -0.032 \\
\hline 1011.4 & 7.976 & 7.966 & -0.021 & -0.015 & -0.039 & 8.101 & 8.092 & -0.024 & -0.017 & -0.035 \\
\hline 1050.7 & 8.002 & & -0.010 & -0.007 & -0.016 & 8.143 & & -0.012 & -0.009 & -0.020 \\
\hline 1052.0 & 7.829 & & -0.010 & -0.007 & -0.017 & 7.975 & & -0.011 & -0.008 & -0.018 \\
\hline 1053.9 & 7.989 & & -0.011 & -0.009 & -0.018 & 8.121 & & -0.013 & -0.009 & -0.022 \\
\hline Average & 7.885 & 7.890 & -0.028 & -0.020 & -0.037 & 8.007 & 8.007 & -0.032 & -0.023 & -0.041 \\
\hline
\end{tabular}

Notes: columns with G are from Grevesse et al. (1990), with B are from Biémont et al. (1990). Column (1) is the wavelength; Cols. (2), (3) $A(\mathrm{~N})$ from 3D model, Cols. (4)-(6) the corresponding NLTE corrections for $S_{\mathrm{H}}=1 / 3,1,0$, respectively; Cols. (7), (8) $A(\mathrm{~N})$ from HM model; Cols. (9)-(11) the corresponding NLTE corrections for $S_{\mathrm{H}}=1 / 3,1,0$, respectively.

always negative, $\approx-0.05$ dex on average, in agreement with the results of Steffen \& Holweger (2002). The total 3D correction, defined as $A(\mathrm{~N})_{3 \mathrm{D}}-A(\mathrm{~N})_{1 \mathrm{D}_{\mathrm{LHD}}}\left(\alpha_{\mathrm{MLT}}=1.0\right)$ is only slightly negative for most of the considered lines. Therefore, the $3 \mathrm{D}$ model provides a slightly smaller abundance (by $\approx-0.01 \mathrm{dex}$ ) than the $1 D_{\text {LHD }}$ model.

The NLTE effects for the analysed lines are small and negative, so that $A(\mathrm{~N})_{\mathrm{LTE}}>A(\mathrm{~N})_{\mathrm{NLTE}}$. The average NLTE correction, for $S_{\mathrm{H}}=1 / 3$, is about $-0.03 \mathrm{dex}$.

\subsection{Estimating the solar metallicity}

Our favoured values for the solar nitrogen abundance, $A(\mathrm{~N})=$ 7.86, and the solar oxygen abundance, $A(\mathrm{O})=8.76$ (Caffau et al. 2008), imply a revision of the solar metallicity $Z$. The two other elements important to the determination of the solar metallicity (in terms of fractional mass) are neon and carbon.

Neon is not measurable in the spectrum of the solar photosphere. The usual procedure is to measure the ratio $\mathrm{Ne} / \mathrm{O}$ in the solar corona, and assume this value to be the same as in the photosphere. We may consider $\mathrm{Ne} / \mathrm{O}=0.15$ (Asplund et al. 2005) or $\mathrm{Ne} / \mathrm{O}=0.18$ (Grevesse \& Sauval 1998), implying $A(\mathrm{Ne})=$ 7.94 and $A(\mathrm{Ne})=8.02$, respectively. By studying $\mathrm{Ne}$ abundances in nearby B-type stars Morel \& Butler (2008) found a mean $A(\mathrm{Ne})=7.97 \pm 0.07$, and suggest that this value should also be representative of the solar Ne abundance. Wang \& Liu (2008) pointed out that the $\mathrm{Ne} / \mathrm{O}$ ratios in $\mathrm{H}$ II regions and planetary nebulae equal on average 0.25 , and suggested that the current estimate of the solar $\mathrm{Ne} / \mathrm{O}$ ratio could be too low. If the solar $\mathrm{Ne} / \mathrm{O}$ ratio was so large, the $\mathrm{Ne}$ abundance would be 8.16. We may therefore consider four possible values of $A(\mathrm{Ne})$, ranging from 7.94 to 8.16 . For the carbon abundance, we have three choices, $A(C)=8.52$ according to Grevesse \& Sauval (1998), $A(\mathrm{C})=8.39$ from Asplund et al. (2005), or $A(\mathrm{C})=8.592$ from Holweger (2001).

The situation is summarised in Table 5, where our $A(\mathrm{~N})$ and $A(\mathrm{O})$ are used with all possible combinations of $A(\mathrm{Ne})$ and $A(\mathrm{C})$ to provide $Z$ and $Z / X$. The metallicity $Z$ spans the range 0.0146 to 0.0167 , while $Z / X$ spans the range 0.0199 to 0.0228 . All these values are considerably higher than the values recommended by
Table 5. Solar metallicity $(Z$ and $Z / X)$ for different choices of $C$ and Ne abundance.

\begin{tabular}{cccc}
\hline \hline $\mathrm{A}(\mathrm{C})$ & 8.39 & 8.52 & 8.59 \\
\hline$A(\mathrm{Ne})$ & & $Z ; Z / X$ \\
\hline 7.94 & $0.0146 ; 0.0199$ & $0.0153 ; 0.0209$ & $0.0158 ; 0.0216$ \\
7.97 & $0.0146 ; 0.0199$ & $0.0154 ; 0.0210$ & $0.0159 ; 0.0218$ \\
8.02 & $0.0148 ; 0.0202$ & $\mathbf{0 . 0 1 5 6} ; \mathbf{0 . 0 2 1 3}$ & $0.0161 ; 0.0220$ \\
8.16 & $0.0154 ; 0.0210$ & $0.0162 ; 0.0221$ & $0.0167 ; 0.0228$ \\
\hline
\end{tabular}

Asplund et al. (2005) $(Z=0.0122$ and $Z / X=0.0165)$, and the increase is mainly driven by our higher oxygen abundance, although the adopted values of $\mathrm{N}, \mathrm{C}$, and $\mathrm{Ne}$ also play a role.

\subsection{Metallicity from helioseismology}

This upward revision alleviates the tension between photospheric abundances and helioseismic data, and the two may essentially agree. Using the solar relative composition of Asplund et al. (2005) and OP opacities, Basu \& Antia (2008) inferred from the depth of the convection zone that $Z / X=0.0218 \pm$ 0.0008 , although the computation should be repeated with our solar composition, but we note that this value is already within the range of the $Z / X$ in Table 5. Using the Asplund et al. (2005) solar composition and one of their test models, Chaplin et al. (2007) derived $Z=0.0161 \pm 0.00008$ from low-degree modes, again in the range of values of Table 5. Using the more extensive set of models obtained from a Monte Carlo simulation, Chaplin et al. (2007) inferred values of between $Z=0.0187$ and $Z=0.0239$. These values are definitely higher than any of the values in Table 5. From the solar sound-speed profile, Antia \& Basu (2006) derive $Z=0.0172 \pm 0.002$. Again, this value is higher than any value in Table 5, even though it is within $2 \sigma$ of the highest value in the table.

\section{Conclusions}

Our recommended value of the solar nitrogen abundance is $A(\mathrm{~N})=7.86 \pm 0.12$, which takes into account $3 \mathrm{D}$ effects and deviations from LTE. This value is between the value inferred by 
Asplund et al. (2005) (7.78 \pm 0.06$)$ and that found by Grevesse \& Sauval (1998) (7.92 \pm 0.06$)$. Our $A(\mathrm{~N})$ is slightly lower, although consistent within errors, with the result found by Holweger $(2001, A(\mathrm{~N})=7.931 \pm 0.111)$.

In the course of our analysis, we found considerable differences between the various solar spectral atlases, introducing additional uncertainties in the abundances derived from individual lines. The disagreement is by far larger than what can be attributed to the noise in the spectra. This fact has been pointed out already in our previous solar abundance studies. We believe that the quality of the available solar spectral atlases, which date back 30 or more years, should be improved by making use of up-to-date technology.

Our most robust estimate of the solar $Z$ is obtained by adopting the abundances of Grevesse \& Sauval (1998) for all elements except for oxygen, nitrogen, and neon, for which we take our own abundances, $A(\mathrm{O})=8.76, A(\mathrm{~N})=7,86$, and $A(\mathrm{Ne})=$ 8.02. The latter results from the ratio $\mathrm{Ne} / \mathrm{O}=0.18$ given by Grevesse \& Sauval (1998), combined with our oxygen abundance. In this way, the resulting solar metallicity is $Z=0.0156$ and $Z / X=0.0213$, respectively.

Pending a new determination of the solar $\mathrm{C}$ abundance, we can say that our new results for $\mathrm{N}$ and $\mathrm{O}$ represent a significant step in forward reconciling photospheric abundances with helioseismic constraints on $Z$.

Acknowledgements. E.C., H.G.L. and P.B. acknowledge support from EU contract MEXT-CT-2004-014265 (CIFIST). The authors thank Katharina Lodders for her input on meteoritic abundances. E.M. is grateful to the Observatoire de Paris and to CNRS for a research stage in Paris during the data analysis procedure of this work.

\section{References}

Anders, E., \& Grevesse, N. 1989, Geochim. Cosmochim. Acta, 53, 197

Antia, H. M., \& Basu, S. 2006, ApJ, 644, 1292

Asplund, M., Grevesse, N., Sauval, A. J., Allende Prieto, C., \& Kiselman, D. 2004, A\&A, 417, 751

Asplund, M., Grevesse, N., \& Sauval, A. J. 2005, Cosmic Abundances as Records of Stellar Evolution and Nucleosynthesis, ASP Conf. Ser., 336, 25

Aufdenberg, J. P., Ludwig, H.-G., \& Kervella, P. 2005, ApJ, 633, 424

Basu, S., \& Antia, H. M. 1997, MNRAS, 287, 189

Basu, S., \& Antia, H. M. 2008, Phys. Rep., 457, 217

Basu, S., Chaplin, W. J., Elsworth, Y., et al. 2007, ApJ, 655, 660

Bevington, P. R., \& Robinson, D. K. 2003, Data reduction and error analysis for the physical sciences, 3rd edn., ed. P. R. Bevington, \& K. D. Robinson (Boston, MA: McGraw-Hill)

Biémont, E., Froese Fischer, C., Godefroid, M., Vaeck, N., \& Hibbert, A. 1990, 3rd International Colloquium of the Royal Netherlands Academy of Arts and Sciences, 59
Caffau, E., \& Ludwig, H.-G. 2007, A\&A, 467, L11

Caffau, E., Ludwig, H.-G., Steffen, M., et al. 2008, A\&A, 488, 1031

Chaplin, W. J., Serenelli, A. M., Basu, S., et al. 2007, ApJ, 670, 872

Delbouille, L., Roland, G., \& Neven, L. 1973, Liege: Universite de Liege, Institut d'Astrophysique

Delbouille, L., Roland, G., Brault, J. W., \& Testerman, L. 1981, Photometric atlas of the solar spectrum from 1850 to $10000 \mathrm{~cm}^{-1}$,

http: //bass2000.obspm. fr/solar_spect.php

Drawin, H. W. 1969, Z. Physik, 225, 483

Freytag, B., Steffen, M., \& Dorch, B. 2002, Astron. Nachr., 323, 213

Freytag, B., Steffen, M., Wedemeyer-Böhm, S., \& Ludwig, H.-G. 2003, CO5BOLD User Manual,

http://www .astro.uu.se/ bf/co5bold_main.html

Grevesse, N., \& Sauval, A. J. 1998, Space Sci. Rev., 85, 161

Grevesse, N., \& Sauval, A. J. 2000, Origin of Elements in the Solar System, Implications of Post-1957 Observations, 261

Grevesse, N., Lambert, D. L., Sauval, A. J., et al. 1990, A\&A, 232, 225

Holweger, H. 1967, ZAp, 65, 365

Holweger, H. 2001, Joint SOHO/ACE workshop, Solar and Galactic Composition, AIP Conf. Proc., 598, 23

Holweger, H., \& Müller, E. A. 1974, Sol. Phys., 39, 19

Holweger, H., Kock, M., \& Bard, A. 1995, A\&A, 296, 233

Houziaux, L. 1961, Zeitschrift fur Astrophysik, 53, 237

Kurucz, R. 1993, SYNTHE Spectrum Synthesis Programs and Line Data. Kurucz CD-ROM No. 18 (Cambridge, Mass.: Smithsonian Astrophysical Observatory)

Kurucz, R. L. 2005a, Mem. Soc. Astron. Ital. Suppl., 8, 189

Kurucz, R. L. 2005b, Mem. Soc. Astron. Ital. Suppl., 8, 14

Lambert, D. L. 1978, MNRAS, 182, 249

Lambert, D. L., \& Swings, J. P. 1967, The Observatory, 87, 113

Ludwig, H.-G., Steffen, M., Freytag, B., et al. 2009, A\&A, in preparation

Moore, C. E. 1945, Contributions from the Princeton University Observatory, 20,1

Moore, C. E., Minnaert, M. G. J., \& Houtgast, J. 1966, National Bureau of Standards Monograph (Washington: US Government Printing Office (USGPO))

Morel, T., \& Butler, K. 2008 [arXiv:0806. 0491]

Mugglestone, D. 1958, MNRAS, 118, 432

Neckel, H., \& Labs, D. 1984, Sol. Phys., 90, 205

Palme, H., \& Jones, A. 2003, Meteorites, Comets and Planets: Treatise on Geochemistry, 1, 41

Ralchenko, Y. 2005, Mem. Soc. Astron. Ital. Suppl., 8, 96,

http://physics.nist.gov/PhysRefData/ASD/index.html

Rentzsch-Holm, I. 1996, A\&A, 305, 275

Sbordone, L. 2005, Mem. Soc. Astron. Ital. Suppl., 8, 61

Sbordone, L., Bonifacio, P., Castelli, F., \& Kurucz, R. L. 2004, Mem. Soc. Astron. Ital. Suppl., 5, 93

Steenbock, W., \& Holweger, H. 1984, A\&A, 130, 319

Steffen, M., \& Holweger, H. 2002, A\&A, 387, 258

Swensson, J. W. 1967, Zeitschrift fur Astrophysik, 66, 156

Swensson, J. W., Benedict, W. S., Delbouille, L., \& Roland, G. 1970, Mémoires of the Société Royale des Sciences de Liège, 5

Wang, W., \& Liu, X. 2008, MNRAS, in press [arXiv:0806.2288]

Wedemeyer, S., Freytag, B., Steffen, M., Ludwig, H.-G., \& Holweger, H. 2004, A\&A, 414, 1121 